\title{
Evaluation of Carpal Tunnel Diagnosis, Management, and Outcome in Primary Health Care Center
}

\author{
Sahar Salem A Albalawi ${ }^{1}$, Ruwaynah Waleed Aziz Alrahman ${ }^{2}$, Abdulrahman Mohsen \\ Althagafi ${ }^{2}$, Abdulaziz Saud Alrahili ${ }^{2}$, Saad Saeed Alqahtani ${ }^{3}$, Salman Mohammed Almutairi ${ }^{3}$, \\ Dhafer Mubarak Alajmi ${ }^{3}$, Zeyad Saad Aljohani ${ }^{4}$, Fahad Abdullah Abualnassr ${ }^{5}$, Ammar \\ Mohammed Sabah ${ }^{5}$ \\ 1- Tabuk university, 2- Umm Al-Qura University, 3- Prince Sattam Bin Abdulaziz University, \\ 4- University of Jeddah, 5- Taibah University
}

\section{Abstract}

Background: Carpal tunnel syndrome (CTS) is one of the commonest conditions encountered in primary care centers as mentioned earlier. CTS of mild to moderate severity can often be effectively treated in a primary care environment. However, many patients being referred immediately to hospital for consideration of surgical decompression.

Objective: In this study, we aim to evaluate the available primary care options and their efficacy in dealing with CTS.

Methods: PubMed database was used for articles selection, and the following keys used in the mesh (("Carpal tunnel syndrome"[Mesh]) AND ("Carpal tunnel syndrome/Management"[Mesh] OR "Carpal tunnel syndrome/Diagnosis"[Mesh] OR "Carpal tunnel syndrome/ Primary Health Care Center)). 5 studies were enrolled according to our inclusion, and exclusion criteria.

Results: Patients successfully treated with wrist splinting alone reported a higher level of satisfaction with their treatment compared to patients who failed wrist splint treatment or had surgical decompressionMechanical traction is associated with fewer surgical interventions compared to care as usual in CTS patients. Reductions in patient-reported symptoms at 6 months' follow-up was similar in both groups.Steroid injection combined with splinting resulted in modestly greater reduction of symptoms, functional recovery, and improvement of nerve function at 12-week follow-up compared to steroid injection alone.

Conclusion: CTS is commonly encountered in primary care. So, a trial of conservative treatment can be done before referral for surgical decompression. Conservative treatment of CTS had shown a clear reduction in hand surgery waitlists, thus reduction in expenditures and costs. Wrist splinting is recommended as first line treatment for patients with symptoms of CTS. Other methods of nonsurgical treatment include steroid injection, osteopathic manipulative therapy, mechanical wrist traction and combining wrist splinting with steroid injection or with NSAIDs use. Referral for surgical decompression may be a more appropriate option when the level of numbness and other symptoms are getting worse, or when conservative management has failed and is likely to lead to dissatisfaction.

Keywords: Carpal Tunnel, Diagnosis, Management, Outcome, Primary Health Care Center

\section{INTRODUCTION}

Carpal tunnel syndrome (CTS) is a neuropathy occur as a result of median nerve compression at the level of the wrist characterized by hand pain, numbness, and tingling in the distribution of the median nerve (thumb, index, middle finger, and the radial side of the ring finger) and a reduction in grip strength and hand function ${ }^{(1-2)}$. The severity of the symptoms can vary between patients into mild, moderate, and severe. Also, most of the time symptoms worse at night ${ }^{(2)}$. CTS can occur in one or both hands, and is either idiopathic (spontaneous) or dynamic (only during certain movements). CTS most of time occur between 40 to 60 years, but also can occur at any age especially in women ${ }^{(1)}$. In USA the prevalence of CTS as estimated to be $4 \%$ of general population and up to $10 \%$ in working population $^{(3)}$. CTS can occur as a result of 
various risk factors such as Obesity, diabetes mellitus, and alcohol abuse ${ }^{(1)}$. Also, occupational factors such as vibration, hand force, and repetition are associated with increased risk for developing CTS ${ }^{(4)}$.

CTS is one of the commonest conditions encountered in primary care centers as mentioned earlier. CTS of mild to moderate severity can often be effectively treated in a primary care environment. However, many patients being referred immediately to hospital for consideration of surgical decompression ${ }^{(5)}$. This review aim to discuss the role of primary care centers in the management of CTS and to evaluate the various available management options that can be done.

\section{METHODOLOGY}

\section{Sample}

PubMed database was used for articles selection, and the following keys used in the mesh "Carpal tunnel syndrome"[Mesh] AND "Carpal tunnel syndrome/Management"'[Mesh] OR "Carpal tunnel syndrome/Diagnosis"[Mesh] OR "Carpal tunnel syndrome/Primary Health Care Center. A total of 46 articles were found, with further restriction by PubMed filters, and reviewing the articles titles and abstracts the final results were 5 articles. Inclusion criteria included the articles were selected based on the relevance to the project which should include one of the following topics, \{Carpal tunnel syndrome, Management, Diagnosis, and Primary Health Care Centers\}. Exclusion criteria excluded all other articles which did not have one of these topics as their primary end point, or repeated studies.

\section{Analysis}

No software was used to analyze the data. The data was extracted based on specific form that contain (Title of the study, name of the author, Objective, Summary, Results, and Outcomes), these data were reviewed by the group members. Double revision of each member's outcomes was applied to ensure the validity of the results.

\section{RESULTS:}

We enrolled 5 studies according to our inclusion, and exclusion criteria described above. All of studies aimed at evaluating the options that are available for the physician in primary care in dealing with patients with CTS symptoms. The studies characteristics are shown in Table 1.

\section{DISCUSSION}

The Carpal tunnel is an osteofibrous outlet, which lies between the flexor retinaculum (FR) and the carpal bones. The roof of this tunnel is the fibrous transverse carpal ligament, which is the intermediate part of the FR. Nine flexor tendons, and their sheath, and the median nerve pass through the tunnel where the nerve enters the tunnel in the midline or slightly radial to it ${ }^{(11)}$. The median nerve gives sensory branches to supply the 3 radial digits and the radial half fourth digit. It also gives the palmar sensory cutaneous branches, which supply the cutaneous skin of the palm, and the thenar (recurrent) motor branch. Carpal tunnel syndrome (CTS) is a neuropathy caused by compression and traction of the median nerve at the level of the carpal tunnel, delimitated by the carpal bones and by the transverse carpal ligament (FR). Another physiological evidence indicates increased pressure within the carpal tunnel due to an inflammation and edema resulting in decreased function of the median nerve at that level $^{(12)}$.

In a retrospective study done on 336 CTS patients in Saudi Arabia Malibary et al. ${ }^{(13)}$, CTS was confirmed in 640 hands (95.23\%) out of 672 with female predominance (86.3\%). The mean age was 52.4 in females and 54.4 years in males. The female to male ratio was $6.3: 1^{(13)}$. Later on, another study, was conducted in another region in Saudi Arabia (Abumunaser LA), showed smaller female to male ratio, 4.9:1. Anyway, these reported ratios are higher than the those in the western 
countries $^{(14)}$. Co-existing conditions were found as diabetes mellitus (35.9\%), hypothyroidism $(14.1 \%)$ and pregnancy $(0.5 \%)^{(13)}$. Goodson et al. ${ }^{(15)}$ conducted a case-control study in USA to investigate the potential risk factors for CTS. They found that occupational repetition, not engaging in

Table 1. Details of the enrolled studies. vigorous exercise, physical activities with wrist strain, poorer physical health, and lower job satisfaction were significantly related to the presence of CTS. Obesity as well was borderline significantly related to the presence of $\mathrm{CTS}^{(15)}$.

\begin{tabular}{|c|c|c|c|c|c|c|}
\hline Study (year) & $\begin{array}{l}\text { Study } \\
\text { Design }\end{array}$ & Country & $\begin{array}{l}\text { Participants } \\
\text { (n) }\end{array}$ & Objective & Duration & $\begin{array}{l}\text { Outcome and } \\
\text { Conclusion }\end{array}$ \\
\hline $\begin{array}{l}\text { B. Povlsen } \\
\text { et al. }\end{array}$ & Cohort & UK & 52 & $\begin{array}{l}\text { To investigate the long-term } \\
\text { outcome of carpal tunnel } \\
\text { syndrome treated with isolated } \\
\text { night wrist splint and the factors } \\
\text { determining the likelihood of } \\
\text { success of this intervention }\end{array}$ & 3 years & $\begin{array}{l}\text { Patients successfully } \\
\text { treated with wrist } \\
\text { splinting alone reported } \\
\text { a higher level of } \\
\text { satisfaction with their } \\
\text { treatment compared to } \\
\text { patients who failed wrist } \\
\text { splint treatment or had } \\
\text { surgical decompression }\end{array}$ \\
\hline$\underset{(7)}{\text { Meems et al. }}$ & RCT & Netherlands & 181 & $\begin{array}{l}\text { To evaluate clinical outcomes } \\
\text { following mechanical traction } \\
\text { in patients with CTS compared } \\
\text { to care as usual such as of a } \\
\text { wrist splint, local corticosteroid } \\
\text { injections or carpal tunnel } \\
\text { release surgery. }\end{array}$ & 2 years & $\begin{array}{l}\text { Mechanical traction is } \\
\text { associated with fewer } \\
\text { surgical interventions } \\
\text { compared to care as } \\
\text { usual in CTS patients. } \\
\text { Reductions in patient- } \\
\text { reported symptoms at } 6 \\
\text { months' follow-up was } \\
\text { similar in both groups. }\end{array}$ \\
\hline$\underset{\text { al. }}{\text { Wang }}$ & $\mathrm{RCT}$ & Taiwan & 52 & $\begin{array}{l}\text { To compare the effectiveness of } \\
\text { local steroid injection plus } \\
\text { splinting to that of local steroid } \\
\text { injection alone using clinical } \\
\text { and electrophysiological } \\
\text { parameters in patients with } \\
\text { carpal tunnel syndrome. }\end{array}$ & $\begin{array}{l}3 \\
\text { months }\end{array}$ & $\begin{array}{l}\text { Steroid injection } \\
\text { combined with splinting } \\
\text { resulted in modestly } \\
\text { greater reduction of } \\
\text { symptoms, functional } \\
\text { recovery, and } \\
\text { improvement of nerve } \\
\text { function at } 12 \text {-week } \\
\text { follow-up compared to } \\
\text { steroid injection alone. }\end{array}$ \\
\hline$\underset{(9)}{\text { Taylor et al. }}$ & Cohort & Canada & 211 & $\begin{array}{l}\text { To determine how frequently } \\
\text { treatments had been offered to } \\
\text { patients with suspected } \\
\text { diagnoses of carpal tunnel } \\
\text { syndrome (CTS) who had been } \\
\text { referred for confirmatory nerve } \\
\text { conduction studies (NCSs) and } \\
\text { to identify potential predictors } \\
\text { of such treatment. }\end{array}$ & $\begin{array}{l}10 \\
\text { months }\end{array}$ & $\begin{array}{l}\text { There was no correlation } \\
\text { found between use of } \\
\text { NSAIDs or wrist splints } \\
\text { and patients' age, sex, } \\
\text { BMI, symptom duration, } \\
\text { symptom or function } \\
\text { scores, or even NCS } \\
\text { results. }\end{array}$ \\
\hline$\underset{(10)}{\text { Jarvik et al. }}$ & RCT & USA & 116 & $\begin{array}{l}\text { To compare surgical versus } \\
\text { multi-modality, non-surgical } \\
\text { treatment for patients with } \\
\text { carpal tunnel syndrome without } \\
\text { denervation. }\end{array}$ & 1 year & $\begin{array}{l}\text { Both groups' symptoms } \\
\text { improved, but surgical } \\
\text { treatment led to better } \\
\text { outcome than did non- } \\
\text { surgical treatment. }\end{array}$ \\
\hline
\end{tabular}


A clear history and targeted examination, which identify standard features and provocative factors, increases the likelihood of a diagnosis. The possibility of CTS should be considered in the presence of symptoms like paresthesia, dull, aching pain, or discomfort in the hand associated with weakness. Female gender, family history, bilateral symptoms, DM, worsening symptoms at night, long duration of symptoms, patient localization of symptoms, hand dominance, symptomatic limb, old age are important factors that can support the diagnosis in the history. The physical examination should start with inspection of the palm. Strong evidence supports Thenar atrophy is strongly associated with ruling-in CTS. However, the absence of the atrophy does not rule out CTS. Upon the local examinations, there are many specific tests but the literature does not support using them independently to diagnose CTS because alone, each has a poor or weak association with ruling-in or ruling-out CTS. Nevertheless, Tinel's Test (repetitive tapping on the carpal tunnel six times), Phalen's Test (patient wrist in full flexion for 60s), and carpal compression test (firm pressure by the physician on the carpal tunnel for 30s) are the most recommended tests to be done in order to support the diagnosis ${ }^{(16-17)}$. In Harrington et al. surveillance criteria for CTS, there should be

Figure 1. Pathway of the patient management. pain or paraesthesia or sensory loss in addition to one of the previously mentioned tests to say it is $\mathrm{CTS}^{(18)}$.

Burton et al. ${ }^{(19)}$ in 2014 developed 8 questions as a diagnosis criteria of CTS (Table 2) and a decision tree (Figure 1) ${ }^{(19)}$.

Table 2. Questions to be asked to a patient presenting with hand or wrist symptoms:

Do you have numbness or tingling in your wrist, hand, or fingers?

Do your symptoms spare your little finger?

Are the symptoms worse at night?

Do the symptoms wake you up at night?

Have you noticed your hand is weak; for example, have you found yourself dropping things?

Do you find shaking your hand, holding your hand or running it under warm water improve your symptoms?

Are the symptoms made worse by activities such as driving, holding a telephone, using vibrating tools, or typing?

Have splints or injections helped with your pain if you have had it in the past?

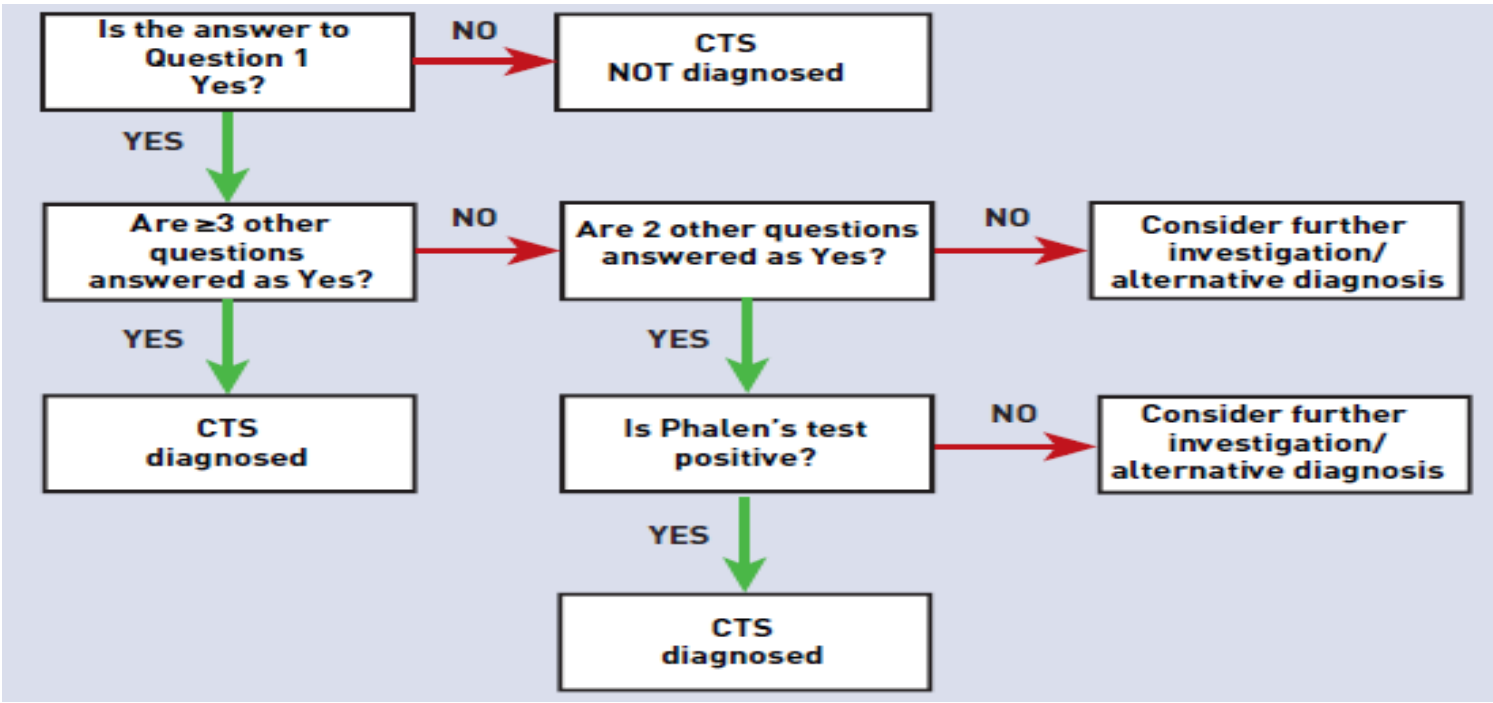


Early cases of CTS mostly present to primary care physicians who can most of the times diagnose CTS, without referral to neither an orthopedician nor a hand surgeon. Similarly, some of the conservative methods of treatments can be started before referral for surgery. Surgical and non-surgical treatment methods improve the symptoms over less than a year, but surgery is associated with better results and a faster improvement of the condition. However, Carpal tunnel release for compression of the median nerve at the wrist is one of the most common and successful procedures in hand surgery. Complications, though rare, are potentially devastating and may include intraoperative technical errors, postoperative infection and pain, and persistent or recurrent symptoms. In $30 \%$ of CTS patients, the symptoms improve spontaneously over 1 year period especially in mild cases. So, the severity is the determinant factor of the approach. In mild to moderate cases of CTS, whereby pain and numbness are intermittent and there is no wasting or weakness of the thenar muscles, non-surgical treatment should be considered ${ }^{(19-20)}$.

The number of surgical interventions performed in the UK for CTS was predicted to increase from 66833 per year in 2015 to 104922 per year by 2030 . Given that a single carpal tunnel release in the UK is projected to cost between $£ 830$ and $£ 2600$, the extrapolated total cost of carpal tunnel release surgery to the UK health budget will exceed $£ 55$ per year. This level of expenditure creates incentives for publicly funded health systems to manage CTS efficiently. In an effort to manage surgery waitlists and reduce costs, retrospective studies in the UK and Australia have examined the effect of alternative care pathways and therapist-led clinics for patients with CTS on surgical waitlists. Despite limited data regarding the cost or clinical effectiveness of conservative interventions, these retrospective case audits have shown a clear reduction in CTS surgery waitlists. The potential benefits of these models of care therefore warrant further investigation ${ }^{(21)}$.
Most of the time, GPs are able to diagnose CTS clinically as mentioned in Povlsen and Raymond paper. Therefore, a GP in primary care can start a trial of non-surgical treatment. Splinting is the most common noninvasive treatment method ${ }^{(22)}$. Povlsen et al. demonstrated that around $70 \%$ of patients with CTS were satisfied following 3 months of wrist splinting alone and half of the patients followed up after around 3 years after splinting treatment had good long-term results, both in terms of symptomatic relief and satisfaction. There does not appear to be a significant difference in pain or numbness between patients who were successfully treated with splints, compared with those who subsequently chose to undergo surgical decompression ${ }^{(6)}$ .Taylor et al. compared between splinting and NSAIDs use as an independent treatment and they were found to be effective among $78 \%$ and $74 \%$ of the patients, respectively ${ }^{(9)}$.

Wrist splinting is recommended as first line treatment for patients with all but the severest symptoms of CTS, in order to protect patients from the potential risks relating to decompression surgery and optimally manage secondary care resources. Those who are suspected to have CTS, should be trialed with wrist splints by their GPs prior to their referral to the specialist clinics. For patients who were happy to continue with the wrist splints after the initial 3 months stage, the chance that this treatment alone would provide a satisfactory outcome could be as high as $43 \%$ after 3 years. However, it is also important to note that, in those who then demonstrate worsening levels of numbness, persevering with wrist splinting is likely to lead to dissatisfaction, and referral for surgical decompression may be a more appropriate option at this stage ${ }^{(6)}$.

When the pain is affecting the quality of life and become unbearable, steroid injection can be suggested. The patient must be informed that steroid injections provide effective temporary relief of symptoms in over $70 \%$ of cases month after injection. However, symptoms tend to recur within a few months. Wang et al. compared in an RCT between 
local steroid injections combined with splinting and local steroid injection alone. The main findings of this study is that steroid injection combined with wrist splinting more effectively improved symptom severity, functional abilities, and nerve function after 12 weeks ${ }^{(8)}$.

It is a simple procedure and can be done by a GP. It should be done by first placing the patient in an upright sitting position with the hand resting on the table, the forearm should be supinated, and the wrist should be placed in slight dorsiflexion. After sterile preparation, a 25-gauge, 1.5-inch needle was inserted inplane immediately superficial and lateral to the ulnar artery and directly above and below the median nerve. It is preferable to be done under ultrasound guidance to avoid injuring the median nerve. The injection fluid contained 1 $\mathrm{ml}$ of $10 \mathrm{mg} \quad(10 \mathrm{mg} / \mathrm{ml})$ triamcinolone acetonide (Shincort) mixed with $1 \mathrm{ml}$ of $2 \%$ lidocaine hydrochloride (Xylocaine) ${ }^{(8)}$.

Osteopathic manipulative therapy (OMT) is one of the recommended treatment methods. It is not commonly used, probably because of a lack of awareness that it could be done in cases of CTS. OMT is an essential component of the conservative management regime for CTS. Various manual techniques have been proved to be effective. ${ }^{(5)}$ Sucher et al. ${ }^{(23)}$ have determined the most effective techniques to elongate the TCL, thereby increasing volume within the carpal tunnel and decreasing pressure on the median nerve. The OMT is described as a powerful and effective form of nonsurgical decompression of the $\mathrm{MN}$ at the CT. It contains many techniques of manipulation such as transverse extension, thenar extension and abduction, hyperextension (wrist and digits), and opponens roll (thenar) ${ }^{(23)}$.

Another promising non-surgical treatment for CTS is mechanical wrist traction. This intervention involves repeated traction movements to the wrist in different positions using gravitational force. The expected mechanism is that traction improves blood microcirculation, reduces edema in the synovial tissue and, therefore, reduces pressure in the carpal tunnel. Meems et al. ${ }^{(7)}$ conducted an RCT in order to evaluate the impact of mechanical traction in patients with CTS. They found mechanical traction can possibly prevent progression of symptoms requiring surgery within 6 months in CTS patients. This approach may result in a substantial reduction in the number of surgeries with similar patientreported symptoms. Mechanical traction may prove to be a more cost-effective intervention for CTS than surgery ${ }^{(7)}$.

This intervention is done by mechanical traction to the wrist is by using weights of between 1 and $18 \mathrm{~kg}$. Patients should receive 12 treatment sessions (twice a week for a period of 6 weeks). One session takes 10 to 15 min per affected hand. The weight was set at 5 $\mathrm{kg}$ for women and $7 \mathrm{~kg}$ for men during the first session. Every following session, the weight should be increased with $1 \mathrm{~kg}$ for women and $2 \mathrm{~kg}$ for men until $10 \mathrm{~kg}$ for women or $13 \mathrm{~kg}$ for men or until the mechanical traction became uncomfortable for the patient. Twelve treatments are considered sufficient for most patients $^{(7)}$.

Patients who are unlikely to respond to conservative management include those who have constant symptoms for more than six months at presentation and those who have already failed to respond to a trial of conservative management. These patients should be referred in order to be considered for surgery.

Hayward et al. ${ }^{(24)}$ prepared a list of suggested reasons for referral. They suggested producing a form of that list in the referral in order for the physician to choose out of them (Table $3)^{(24)}$.

Table 3. List of suggested reasons for referral.

The patient has constant symptoms. At presentation his her symptoms had already been present for more than 6 months. Helshe is found to have thenar wasting. His ther condition makes it impossible to work and threatens employment. 
The patient is over 50 years old and Phalen's test becomes positive in less than 30 seconds.

The patient has failed to respond to a 6 month trial of conservative management.

The patient responded to a 6 month trial of conservative management, but their symptoms have now returned.

The patient understands that surgery may be necessary.

\section{CONCLUSION}

CTS is commonly encountered in primary care. So, a trial of conservative treatment can be done before referral for surgical decompression. Conservative treatment of CTS had shown a clear reduction in hand surgery waitlists, thus reduction in expenditures and costs. Wrist splinting is recommended as first line treatment for patients with symptoms of CTS. Other methods of non-surgical treatment include steroid injection, osteopathic manipulative therapy, mechanical wrist traction and combining wrist splinting with steroid injection or with NSAIDs use. Referral for surgical decompression may be a more appropriate option when the level of numbness and other symptoms are getting worse, or when conservative management has failed and is likely to lead to dissatisfaction.

\section{REFERENCES}

1. Ibrahim I (2012): Carpal Tunnel Syndrome: A Review of the Recent Literature. The Open Orthopaedics Journal, 6(1):69-76.

2. Dawson D (1993): Entrapment Neuropathies of the Upper Extremities. New England Journal of Medicine, 329(27): 2013-2018.

3. Atroshi I (1999): prevalence of Carpal Tunnel Syndrome in a General Population. JAMA., 282(2): 153.

4. Ibrahim I (2012): Carpal Tunnel Syndrome: A Review of the Recent Literature. The Open Orthopaedics Journal, 6(1):69-76.
5. Dawson D(1993): Entrapment Neuropathies of the Upper Extremities. New England Journal of Medicine, 329(27): 2013-2018.

6. Atroshi I (1999): revalence of Carpal Tunnel Syndrome in a General Population. Jama., 282(2): 153.

7. Spahn G, Wollny J, Hartmann B et al. (2012): Metaanalysis for the Evaluation of Risk Factors for Carpal Tunnel Syndrome (CTS) Part II. Occupational Risk Factors. Zeitschrift Für Orthopädie Und Unfallchirurgie, 150(5): 516-24

8. Schreiber A(2014): Two Novel Nonsurgical Treatments of Carpal Tunnel Syndrome. Physical Medicine and Rehabilitation Clinics of North America, 25(2): 249-64.

9. Povlsen Bo, Bashir M, Wong F et al. (2013): Long-term Result and Patient Reported Outcome of Wrist Splint Treatment for Carpal Tunnel Syndrome." Journal of Plastic Surgery and Hand Surgery, 48(3): 175-78.

10. Margreet M, Spek V, Kop WJ et al. (2017): Mechanical Wrist Traction as a Non-invasive Treatment for Carpal Tunnel Syndrome: A Randomized Controlled Trial.Trials, 18: 464.

11. Jia-Chi W, Kwong-Kum L, KonPing L et al. (2017): Efficacy of Combined Ultrasound-Guided Steroid Injection and Splinting in Patients With Carpal Tunnel Syndrome. Archives of Physical Medicine and Rehabilitation, 98(5): 947-56.

12. Taylor-Gjevre R, Gjevre J, Strueby L et al. (2007): Treatments for carpal tunnel syndrome: Who does what, when and why? Canadian Family Physician, 53(7):1186-1190.

13. Jeffrey J, Comstock B, Kliot $M$ et al. (2009): Surgery versus Nonsurgical Therapy for Carpal Tunnel Syndrome: A Randomised Parallelgroup Trial. The Lancet, 374(9695):1074-081.

14. Macdermid JC (2004): Clinical and Electrodiagnostic Testing of Carpal Tunnel Syndrome: A Narrative Review. J Orthop Sports Phys Ther. ,34(10):565-588. 
15. Aboonq MS (2015): Pathophysiology of carpal tunnel syndrome. Neurosciences, 20(1):4-9.

16. Malibary HM, Al-Najjar A, Yassen DM et al. (2013): Clinical Profile of Carpal Tunnel Syndrome in a Teaching Hospital. Pak J Med Sci., 29(1): 119-121.

17. Abumunaser

A(2012):

Demographic pattern of carpal tunnel syndrome in western Saudi Arabia. Neurosciences, 17: 44-47.

18. Goodson JT, Scott D, Anthony J et al. (2014): Occupational and Biopsychosocial Risk Factors for Carpal Tunnel Syndrome. Journal of Occupational and Environmental Medicine, 56(9): 965-972.

19. Graham B, Allan E, Robert A et al. (2016): The American Academy of Orthopaedic Surgeons EvidenceBased Clinical Practice Guideline on. The Journal of Bone and Joint Surgery, 98(20):1750-1754.

20. Burke F, Ellis J, McKenna $\mathrm{H}$ et al. (2003): Primary Care Management of Carpal Tunnel Syndrome. Postgraduate Medical Journal, 79(934):433-437.

21. Harrington JM, Carter JT, Birrell L et al. (1998): Surveillance Case Definitions for Work Related Upper Limb Pain Syndromes. Occupational and Environmental Medicine, 55(4):264-271.
22. Burton C, Linda S, Graham D (2014): Diagnosing and Managing Carpal Tunnel Syndrome in Primary Care. Br J Gen Pract., 64(622): 262263.

23. Padua L, Aprile I, Caliandro $P$ (2002): Carpal Tunnel Syndrome in Pregnancy: Multiperspective Followup of Untreated Cases. Neurology, 59(10): 1643-1646.

24. Lewis K, Leo Ross M, Coppieters B (2016): Education, Night Splinting and Exercise versus Usual Care on Recovery and Conversion to Surgery for People Awaiting Carpal Tunnel Surgery: A Protocol for a Randomised Controlled Trial. BMJ Open, doi:10.1136/bmjopen-2016-012053.

25. Povlsen B, Raymond A (2012): GP Diagnostics and Splint Treatment of Carpal Tunnel Syndrome and Trapezio metacarpal Arthritis. The Bulletin of the Royal College of Surgeons of England, 94 (8): 1-2.

26. Sucher BM, Hinrichs RN (1998): Manipulative treatment of carpal tunnel syndrome: biomechanical and osteopathic intervention to increase the length of the transverse carpal ligament. J Am Osteopath Assoc., 98:679-686.

27. Hayward A C (2002): Primary Care Referral Protocol for Carpal Tunnel Syndrome. Postgraduate Medical Journal, $\quad$ 78(917):149-152. 